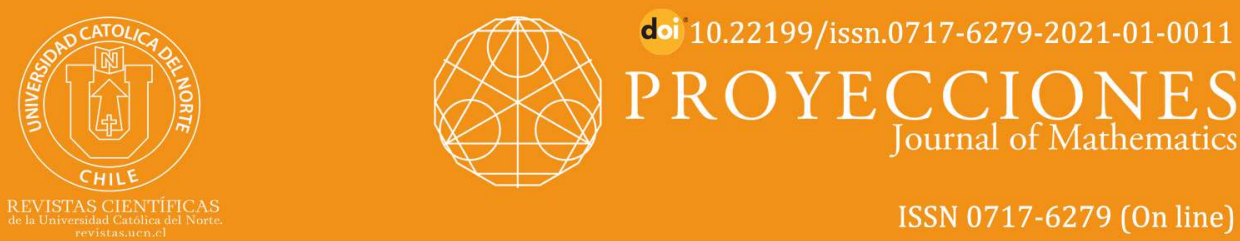

\title{
Minimal and maximal solutions to first-order differential equations with piecewise constant generalized delay
}

Kuo-Shou Chiu ${ }^{1}$ (D) orcid.org/0000-0002-3823-5898

${ }^{1}$ Universidad Metropolitana de Ciencias de la Educación, Depto. de Matemática, Santiago, Chile. ㅈschiu@umce.cl

\section{Abstract:}

In this paper we employ the method of maximal and minimal solutions coupled with comparison principles and the monotone iterative technique to obtain results of existence and approximation of solutions for differential equations with piecewise constant delay of generalized type (DEPCAG).

Keywords: Piecewise constant arguments; Monotone iterative method; Comparison results; Maximal and minimal solutions.

MSC (2020): 34A12, 34A36, 34K12, 26D10.

\section{Cite this article as (IEEE citation style):}

K.-S. Chiu, "Minimal and maximal solutions to first-order differential equations with piecewise constant generalized delay", Proyecciones (Antofagasta, On line), vol. 40, no. 1, pp. 175-186, 2021, doi: 10.22199/issn.0717-6279-2021-01-0011

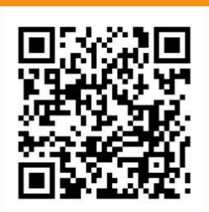

Article copyright: (C) 2021 Kuo-Shou Chiu. This is an open access article distributed under the terms of the Creative Commons License, which permits unrestricted use and distribution provided the original author and source are credited. 


\section{Introduction}

The study of differential equations with piecewise constant arguments has been treated widely in the literature. This type of equation, in which techniques of differential and difference equations are combined, models, among others, some biological phenomena (see $[3,15]$ and references therein), the stabilization of hybrid control systems with feedback discrete controller [16], or damped oscillators [7]. The first studies in this field have been given in $[4,20]$, after this, some papers related with stability, oscillation properties and existence of periodic solutions have been treated by several authors (see $[5,8,9,14,22]$ for details).

It is well known that the theory of differential inequalities for initialvalue problems has been very useful in the theory of differential equations $[18,21]$. Existence of extremal solutions for a variety of nonlinear differential equations is studied by a combined approach of the method of maximal and minimal solutions and the monotone iterative technique [17]. It is natural to extend this useful method to retarded differential equations.

In this paper we continue the investigation of differential equations with piecewise constant argument of generalized type (DEPCAG) originated in $[2,19]$, using differential inequalities. These equations are closely related to impulse and loaded equations and, especially, to difference equations of a discrete argument. They have the structure of continuous dynamical systems within intervals of certain length. Continuity of a solution at a point joining any two consecutive intervals then implies recursion relations for the solution at such points. The results obtained in $[2,5,6,7,8,9,10,11,12,13,19]$ show that all types of DEPCAG share similar characteristics.

First of all, it is natural to pose the initial-value problem for such equations not on an interval but at a number of individual points. Secondly, two-sided solutions exist for all types of DEPCAG. Finally, since DEPCAG combine the features of both differential and difference equations, their asymptotic behavior as $t \rightarrow \infty$ resembles the solutions growth of differential equations in some cases, while in others it inherits the properties of difference equations.

The paper is organized as follows, in Section 2 we present the main tools that we will use in the rest of the paper. Section 3 is devoted to obtain the unique solution of the associated linear problem, from which we derive comparison results.

In Section 4, we present results concerning the existence of extremal 
solutions and the uniqueness of solution in the presence of lower and upper solutions.

\section{Preliminaries}

Let $\mathbf{Z}$ and $\mathbf{R}$ be the sets of integer and real numbers, respectively. Fix a real sequence $t_{i}, i \in \mathbf{Z}$, is a strictly ordered sequence of real number, such that $t_{i}<t_{i+1}, t_{i} \rightarrow \infty$ as $i \rightarrow \infty$. Let $\beta: \mathbf{R} \rightarrow \mathbf{R}$ be a step function given by $\beta(t)=t_{i}$ for $t \in I_{i}=\left[t_{i}, t_{i+1}\right)$ and there exists a positive real number $\vartheta$ such that $t_{i+1}-t_{i}=\vartheta_{i} \leq \vartheta, i \in \mathbf{Z}$. For every $t \in \mathbf{R}$, let $i=i(t) \in \mathbf{Z}$ be the unique integer such that $t \in I_{i}=\left[t_{i}, t_{i+1}\right)$. To simplify notations, we will denote by $\Theta$ that is a space of strictly ordered sequences $\left\{t_{i}\right\} \in \mathbf{R}, i \in \mathbf{Z}$, such that $\left|t_{i}\right| \rightarrow \infty$ if $|i| \rightarrow \infty$.

We study, in this paper, the existence of minimal and maximal solutions for the differential equation with piecewise constant generalized delay of the form

$$
x^{\prime}(t)=f(t, x(t), x(\beta(t))), \quad x(\tau)=c_{0},
$$

where $\beta(t)=t_{i}$ if $t \in I_{i}=\left[t_{i}, t_{i+1}\right), i \in \mathbf{Z}$, is the identification function and $f$ is continuous on $\mathbf{R} \times \mathbf{R} \times \mathbf{R}$.

We introduce the following definition.

Definition 1. A function $x$ is a solution of the DEPCAG (2.1) in $\mathbf{R}$ if i) $x$ is continuous on $\mathbf{R}$.

ii) The derivative $x^{\prime}(t)$ exists at each point $t \in \mathbf{R}$ with the possible exception of the points $t_{i} \in \mathbf{R}, i \in \mathbf{Z}$, where the one-side derivatives exist.

iii) The DEPCAG (2.1) is satisfied for $x$ on each interval $\left(t_{i}, t_{i+1}\right), i \in \mathbf{Z}$, and it holds for the right derivative at the points $t_{i}, i \in \mathbf{Z}$.

We will use, in the discussion of the problem, several properties of the function $\lambda_{a, b}(t)=\lambda(t):[\tau, \infty) \rightarrow \mathbf{R}$ defined by

$$
\lambda_{a, b}(t)=\lambda(t)=e^{a t}+\frac{b}{a}\left(e^{a t}-1\right) .
$$

It is easy to verify that $\lambda_{a, b}^{\prime}(t)=(a+b) e^{a t}$ for all $t \in[\tau, \infty)$ and, as an immediate consequence, is strictly monotone increasing on $\mathbf{R}$ for $a+b>0$ and strictly monotone decreasing on $\mathbf{R}$ if $a+b<0$. Obviously, when $a=-b$, is a constant function equal to 1 . Clearly, for all $a, b \in \mathbf{R}$, we have that $\lambda(0)=1$. 


\section{The linear problem: comparison results}

In this section we analyze the following linear differential equation with a general piecewise constant delay

$$
y^{\prime}(t)=a y(t)+b y(\beta(t))+g(t), \quad y(\tau)=c_{0},
$$

where $\tau \in \Theta, a$ and $b$ are real constants and $g(t)$ is continuous function on $[\tau, \infty)$. At a first moment we prove that this problem has a unique solution.

Theorem 1. The linear initial value problem with the DEPCAG (3.1) has on $[\tau, \infty)$ a unique solution given by

$$
y(t)=\mu(t)\left(c_{0} \mu_{i(\tau)}^{i(t)-1}+S\right)+\int_{\beta(t)}^{t} e^{a \cdot(t-s)} g(s) d s,
$$

where

$$
S=\sum_{j=i(\tau)}^{i(t)-1} \mu_{j}^{i(t)-1} \int_{t_{j}}^{t_{j+1}} e^{a \cdot\left(t_{j+1}-s\right)} g(s) d s
$$

$g(t)$ is continuous on $[\tau, \infty), \tau \in \Theta$, and

$\mu(t)=\lambda(t-\beta(t)), \quad \mu_{j}^{i}=\prod_{k=j}^{i} \lambda\left(t_{k+1}-t_{k}\right)=\prod_{k=j}^{i} \lambda\left(\vartheta_{k}\right), \quad$ and $\quad i \geq j>i(\tau)$.

Proof. Let $y_{n}(t)$ be solutions of the DEPCAG (3.1) in the interval $\left[t_{n}, t_{n+1}\right)$, satisfying the condition $y_{n}\left(t_{n}\right)=y_{n}$, for $n \in\{i(\tau)+j\}_{j \in \mathbf{N}}$.

Then we have

$$
y_{n}(t)=y_{n} \lambda\left(t-t_{n}\right)+\int_{t_{n}}^{t} e^{a \cdot(t-s)} g(s) d s,
$$

where, $\lambda$ is given in (2.2). From (3.3) and $t \rightarrow t_{n+1}$, we obtain

$$
y_{n+1}=y_{n} \lambda\left(\vartheta_{n}\right)+\int_{t_{n}}^{t_{n+1}} e^{a \cdot\left(t_{n+1}-s\right)} g(s) d s .
$$

Applying (3.4) repeatedly for $y_{n-1}, y_{n-2}, \ldots, y_{i(\tau)+1}$ yields

$$
\begin{aligned}
y_{n+1} & =\left(\prod_{j=i(\tau)+1}^{n} \lambda\left(\vartheta_{j}\right)\right) y_{i(\tau)+1} \\
& +\sum_{j=i(\tau)+1}^{n} \prod_{k=j+1}^{n} \lambda\left(\vartheta_{k}\right) \times \int_{t_{j}}^{t_{j+1}} e^{a \cdot\left(t_{j+1}-s\right)} g(s) d s \\
& +\int_{t_{n}}^{t_{n+1}} e^{a \cdot\left(t_{n+1}-s\right)} g(s) d s .
\end{aligned}
$$


From (3.3), one gets

$$
y\left(t_{i(\tau)+1}\right)=y(\tau) \lambda\left(t_{i(\tau)+1}-\tau\right)+\int_{\tau}^{t_{i(\tau)+1}} e^{a \cdot\left(t_{i(\tau)+1}-s\right)} g(s) d s .
$$

Use (3.3), (3.5), (3.6) and $\tau \in \Theta$ to obtain finally

$$
\begin{aligned}
y_{n}(t) & =\lambda\left(t-t_{n}\right)\left(\prod_{j=i(\tau)}^{n-1} \lambda\left(\vartheta_{j}\right)\right) y(\tau) \\
& +\lambda\left(t-t_{n}\right)\left(\prod_{j=i(\tau)}^{n-1} \lambda\left(\vartheta_{j}\right)\right) \int_{\tau}^{t_{i(\tau)}} e^{a \cdot(\tau-s)} g(s) d s \\
& +\lambda\left(t-t_{n}\right) \sum_{j=i(\tau)}^{n-1} \prod_{k=j+1}^{n-1} \lambda\left(\vartheta_{k}\right) \int_{t_{j}}^{t_{j+1}} e^{a \cdot\left(t_{j+1}-s\right)} g(s) d s \\
& +\int_{t_{n}}^{t} e^{a \cdot\left(t_{n}-s\right)} g(s) d s,
\end{aligned}
$$

from where (3.2) follows.

Note that (3.2) is obtained with the implicit assumption $a \neq 0$. If $a=0$, then the unique solution of (3.1) is given by

$$
(3.8) y(t)=(1+b(t-\beta(t)))\left\{c_{0} \prod_{j=i(\tau)}^{i(t)-1}\left(1+b \vartheta_{j}\right)+\hat{S}\right\}+\int_{\beta(t)}^{t} g(s) d s
$$

where

$$
\hat{S}=\sum_{j=i(\tau)}^{i(t)-1}\left(\prod_{k=j}^{i(t)-1}\left(1+b \vartheta_{k}\right)\right) \int_{t_{j}}^{t_{j+1}} g(s) d s,
$$

which is the limiting case of (3.2) as $a \rightarrow 0$.

Remark 1. Theorem 1 generalizes the linear differential equation with a piecewise constant delay considered by Aftabizadeh and Wiener [1, pp.190] with $\beta(t)=[t]$.

In order to obtain existence results for the DEPCAG (2.1) we will use monotone iterative techniques. It is very well known that a fundamental tool to treat this kind of problems consists in maximum principles of the linear operator studied above. From the form of the solution of the linear DEPCAG (3.1) we can deduce the following. 
Lemma 1. Suppose that $y \in C[[\tau, \infty), \mathbf{R}]$, and the derivative $y^{\prime}(t)$ exists at each point $t \in[\tau, \infty)$, with the possible exception of the points $t_{i} \in[\tau, \infty)$, $i \in \mathbf{Z}$, where one-sided derivatives exist. Assume that

$$
y^{\prime}(t) \leq a y(t)+b y(\beta(t)), \quad y(\tau) \leq 0,
$$

where $a$ and $b$ are constants such that

$$
b \geq-\frac{a e^{a \vartheta_{j}}}{e^{a \vartheta_{j}}-1}, \quad j \geq i(\tau)
$$

Then $y(t) \leq 0$ on $[\tau, \infty)$.

Proof. For $t \in\left[t_{n}, t_{n+1}\right)$, for $n \geq i(\tau)$, consider

$$
y_{n}^{\prime}(t) \leq a y_{n}(t)+b y_{n}\left(t_{n}\right), \quad y_{n}\left(t_{n}\right) \leq 0 .
$$

From this and (3.10) we find that $y_{n}(t) \leq 0, t \in\left[t_{n}, t_{n+1}\right)$. This implies, because of the continuity, $y(t) \leq 0$ for $t \geq \tau$.

Remark 2. Lemma 1 generalizes the corresponding inequality considered by Aftabizadeh and Wiener [1, Lemma 3.2] with $\beta(t)=[t]$.

Note that if $a=0$, from the proof of Lemma 1 , we can obtain the following Corollary.

Corollary 1. Suppose that $y \in C[[\tau, \infty), \mathbf{R}]$, and the derivative $y^{\prime}(t)$ exists at each point $t \in[\tau, \infty)$, with the possible exception of the points $t_{i} \in$ $[\tau, \infty), i \in \mathbf{Z}$, where one-sided derivatives exist. Assume that

$$
y^{\prime}(t) \leq b y(\beta(t)), \quad y(\tau) \leq 0,
$$

where $b$ is a real constant such that

$$
b \geq-\frac{1}{\vartheta_{j}}, \quad j \geq i(\tau)
$$

Then $y(t) \leq 0$ on $[\tau, \infty)$. 


\section{Minimal and maximal solutions}

In this section we apply the monotone method to prove the existence of minimal and maximal solutions for DEPCAG, so we recall the DEPCAG (2.1):

$$
x^{\prime}(t)=f(t, x(t), x(\beta(t))), \quad x(\tau)=c_{0},
$$

where $\tau \in \Theta$ and $f \in C[[\tau, \infty) \times \mathbf{R} \times \mathbf{R}, \mathbf{R}]$. This constructive method yields monotone sequences that converge to solutions of (4.1). Since each member of these sequences happens to be the solution of a linear delay differential equation which can be computed explicitly, the advantage and importance of the technique need no special emphasis. For more details of the monotone iterative technique, the reader should refer to [4, 23].

We introduce the following definitions.

Definition 2. We say that $\underline{\alpha}(t)$ is a lower solution of the DEPCAG (4.1) if the following inequalities hold

$$
\underline{\alpha}(t) \leq f(t, \underline{\alpha}(t), \underline{\alpha}(\beta(t))), \quad \underline{\alpha}(\tau) \leq c_{0} .
$$

In an analogous way, we say that $\bar{\alpha}(t)$ is an upper solution of the DEPCAG (4.1) if the following inequalities hold

$$
\bar{\alpha}(t) \geq f(t, \bar{\alpha}(t), \bar{\alpha}(\beta(t))), \quad \bar{\alpha}(\tau) \geq c_{0} .
$$

Theorem 2. Let $\underline{\alpha}(t)$ and $\bar{\alpha}(t)$ be lower and upper solutions of the DEPCAG (4.1) such that $\underline{\alpha}(t) \leq \bar{\alpha}(t)$ on $I=[\tau, \infty)$. Suppose that

$$
f(t, u, v)-f(t, x, y) \geq a \cdot(u-x)+b \cdot(v-y), \quad t \geq \tau,
$$

for $\underline{\alpha}(t) \leq x(t) \leq u(t) \leq \bar{\alpha}(t), \underline{\alpha}(t) \leq y(t) \leq v(t) \leq \bar{\alpha}(t)$, and

$$
b \geq-\frac{a e^{a \vartheta_{j}}}{e^{a \vartheta_{j}}-1}, \quad j \geq i(\tau) .
$$

Then there exist monotone sequences $\left\{\underline{\alpha}_{m}(t)\right\}$ and $\left\{\bar{\alpha}_{m}(t)\right\}$ with $\underline{\alpha}_{0}(t)=$ $\underline{\alpha}(t), \bar{\alpha}_{0}(t)=\bar{\alpha}(t)$ such that $\underline{\alpha}_{m}(t) \rightarrow \underline{\alpha}_{*}(t), \bar{\alpha}_{m}(t) \rightarrow \bar{\alpha}_{*}(t)$ as $m \rightarrow \infty$ monotonically on $I$, and $\bar{\alpha}_{*}(t), \underline{\alpha}_{*}(t)$ are minimal and maximal solutions of the DEPCAG (4.1). 
Proof. For any $\eta \in C[[\tau, \infty), \mathbf{R}]$ such that $\underline{\alpha}(t) \leq \eta(t) \leq \bar{\alpha}(t)$, we consider the linear DEPCAG (3.1) with

$$
g(t)=f(t, \eta(t), \eta(\beta(t)))-a \eta(t)-b \eta(\beta(t)),
$$

i.e.,

$$
y^{\prime}(t)=a y(t)+b y(\beta(t))+f(t, \eta(t), \eta(\beta(t)))-a \eta(t)-b \eta(\beta(t)),
$$

with $y(\tau)=c_{0}$. It is clear that for every such $\eta$, by Theorem 1 , there exists a unique solution $y(t)$ of the linear DEPCAG $(4.4)$ on $[\tau, \infty)$. Define a mapping $\Im$ by $\Im \eta=y$, where $y$ is the unique solution of the linear DEPCAG (4.4). This mapping will be used to define the sequences $\left\{\underline{\alpha}_{m}(t)\right\}$ and $\left\{\bar{\alpha}_{m}(t)\right\}$. We complete the proof by four steps:

Step 1. We claim that $\underline{\alpha} \leq \Im \underline{\alpha}$ and $\Im \bar{\alpha} \leq \bar{\alpha}$.

Let $\underline{\alpha}_{1}=\Im \underline{\alpha}$, then $\underline{\alpha}_{1}$ satisfies

$$
\underline{\alpha}_{1}^{\prime}(t)-a \underline{\alpha}_{1}(t)-b \underline{\alpha}_{1}(\beta(t))=f(t, \underline{\alpha}(t), \underline{\alpha}(\beta(t)))-a \underline{\alpha}(t)-b \underline{\alpha}(\beta(t)) .
$$

Let

$$
p(t)=\underline{\alpha}(t)-\underline{\alpha}_{1}(t) .
$$

On each unit interval $\left[t_{n}, t_{n+1}\right), n \geq i(\tau)$, we have

$$
p_{n}(t)=\underline{\alpha}_{n}(t)-\underline{\alpha}_{n 1}(t),
$$

where $\underline{\alpha}_{n 1}(t)$ is the solution of the DEPCAG (4.4) on $\left[t_{n}, t_{n+1}\right)$ with $\eta=\underline{\alpha}_{n}$ and $\underline{\alpha}_{n 1}\left(t_{n}\right)=\hat{c}_{n}$. Then from $p_{n}^{\prime}(t)=\underline{\alpha}_{n}^{\prime}(t)-\underline{\alpha}_{n 1}^{\prime}(t)$ and the fact that $\underline{\alpha}_{n}(t)$ is the lower solution of the DEPCAG (4.1), i.e.,

$$
\underline{\alpha}_{n}^{\prime}(t) \leq f\left(t, \underline{\alpha}_{n}(t), \underline{\alpha}_{n}\left(t_{n}\right)\right)
$$

we have

$$
p_{n}^{\prime}(t) \leq a p_{n}(t)+b p_{n}\left(t_{n}\right), \quad p_{n}\left(t_{n}\right) \leq 0 .
$$

From (4.3) and Lemma 1 , we obtain $p_{n}(t) \leq 0$ for $\left[t_{n}, t_{n+1}\right), n \geq i(\tau)$, and thus $p(t) \leq 0$ on $[\tau, \infty)$, which shows that $\underline{\alpha} \leq \Im \underline{\alpha}$. Similarly, we can show that $\Im \bar{\alpha} \leq \bar{\alpha}$. 
Step 2. We show that $\Im \eta_{1} \leq \Im \eta_{2}$ if $\underline{\alpha} \leq \eta_{1} \leq \eta_{2} \leq \bar{\alpha}$.

Let $\eta_{1}^{*}=\Im \eta_{1}, \eta_{2}^{*}=\Im \eta_{2}$ and $p=\eta_{1}^{*}-\eta_{2}^{*}$, then for $\left[t_{n}, t_{n+1}\right)$, and by (4.2), we obtain

$$
\begin{aligned}
p_{n}^{\prime}(t)= & f\left(t, \eta_{1}(t), \eta_{1}(\beta(t))\right)-f\left(t, \eta_{2}(t), \eta_{2}(\beta(t))\right) \\
& +a \cdot\left(\eta_{1}^{*}(t)-\eta_{1}(t)\right)-a \cdot\left(\eta_{2}^{*}(t)-\eta_{2}(t)\right) \\
& +b \cdot\left(\eta_{1}^{*}(\beta(t))-\eta_{1}(\beta(t))\right)-b \cdot\left(\eta_{2}^{*}(\beta(t))-\eta_{2}(\beta(t))\right) \\
\leq & -a \cdot\left(\eta_{2}(t)-\eta_{1}(t)\right)-b \cdot\left(\eta_{2}(\beta(t))-\eta_{1}(\beta(t))\right) \\
& +a \cdot\left(\eta_{1}^{*}(t)-\eta_{1}(t)\right)-a \cdot\left(\eta_{2}^{*}(t)-\eta_{2}(t)\right) \\
& +b \cdot\left(\eta_{1}^{*}(\beta(t))-\eta_{1}(\beta(t))\right)-b \cdot\left(\eta_{2}^{*}(\beta(t))-\eta_{2}(\beta(t))\right) \\
\leq & a \cdot\left(\eta_{1}^{*}(t)-\eta_{2}^{*}(t)\right)+b \cdot\left(\eta_{1}^{*}(\beta(t))-\eta_{2}^{*}(\beta(t))\right)=a p_{n}(t)+b p_{n}\left(t_{n}\right),
\end{aligned}
$$

with $p_{n}\left(t_{n}\right) \leq 0$. Still by (4.3) and Lemma 1 , we obtain $p_{n}(t) \leq 0$ for $\left[t_{n}, t_{n+1}\right), n \geq i(\tau)$, and thus $p(t) \leq 0$ on $[\tau, \infty)$, which implies $\Im \eta_{1} \leq \Im \eta_{2}$.

Step 3. We prove that the DEPCAG (4.1) have solutions.

Let $\underline{\alpha}_{i}=\Im \underline{\alpha}_{i-1}, \bar{\alpha}_{i}=\Im \bar{\alpha}_{i-1}, n=1,2,3, \ldots$. Following the first two s, we have

$\underline{\alpha}(t)=\underline{\alpha}_{0}(t) \leq \underline{\alpha}_{1}(t) \leq \cdots \leq \underline{\alpha}_{n}(t) \leq \cdots \leq \bar{\alpha}_{n}(t) \leq \cdots \leq \bar{\alpha}_{1}(t) \leq \bar{\alpha}_{0}(t)=\bar{\alpha}(t)$.

Obviously, each $\underline{\alpha}_{i}, \bar{\alpha}_{i}(i=1,2,3, \ldots)$ satisfies

$\underline{\alpha}_{i}^{\prime}(t)-a \underline{\alpha}_{i}(t)-b \underline{\alpha}_{i}(\beta(t))=f\left(t, \underline{\alpha}_{i-1}(t), \underline{\alpha}_{i-1}(\beta(t))\right)-a \underline{\alpha}_{i-1}(t)-b \underline{\alpha}_{i-1}(\beta(t))$, $\bar{\alpha}_{i}^{\prime}(t)-a \bar{\alpha}_{i}(t)-b \bar{\alpha}_{i}(\beta(t))=f\left(t, \bar{\alpha}_{i-1}(t), \bar{\alpha}_{i-1}(\beta(t))\right)-a \bar{\alpha}_{i-1}(t)-b \bar{\alpha}_{i-1}(\beta(t))$, with $\underline{\alpha}(\tau)=\bar{\alpha}(\tau)=c_{0}$. Therefore there exist $\underline{\alpha}_{*}$ and $\bar{\alpha}_{*}$ such that

$$
\lim _{i \rightarrow \infty} \underline{\alpha}_{i}=\underline{\alpha}_{*}(t), \lim _{i \rightarrow \infty} \bar{\alpha}_{i}=\bar{\alpha}_{*}(t)
$$

uniformly on $[\tau, \infty)$. Clearly, $\underline{\alpha}_{*}$ and $\bar{\alpha}_{*}$ satisfy the DEPCAG (4.1).

Step 4. We prove $\underline{\alpha}_{*}$ and $\bar{\alpha}_{*}$ are extreme solutions of the DEPCAG (4.1). Let $y(t)$ be any solution of the DEPCAG (4.1), which satisfies $\underline{\alpha}(t) \leq y(t) \leq \bar{\alpha}(t), t \in[\tau, \infty)$. Also suppose there exists a positive integer $n$ such that for $t \in[\tau, \infty), \underline{\alpha}_{n}(t) \leq y(t) \leq \bar{\alpha}_{n}(t)$. 
Setting $p(t)=\underline{\alpha}_{n+1}(t)-y(t)$, then for $t \in[\tau, \infty)$, and by (4.2), we obtain

$$
\begin{aligned}
p^{\prime}(t)= & f\left(t, \underline{\alpha}_{n}(t), \underline{\alpha}_{n}(\beta(t))\right)+a \cdot\left(\underline{\alpha}_{n+1}(t)-\underline{\alpha}_{n}(t)\right) \\
& +b \cdot\left(\underline{\alpha}_{n+1}(\beta(t))-\underline{\alpha}_{n}(\beta(t))\right)-f(t, y(t), y(\beta(t))) \\
= & a \cdot\left(\underline{\alpha}_{n+1}(t)-y(t)\right)+b \cdot\left(\underline{\alpha}_{n+1}(\beta(t))-y(\beta(t))\right) \\
& +f\left(t, \underline{\alpha}_{n}(t), \underline{\alpha}_{n}(\beta(t))\right)-f(t, y(t), y(\beta(t))) \\
& -a \cdot\left(\underline{\alpha}_{n}(t)-y(t)\right)-b \cdot\left(\underline{\alpha}_{n}(\beta(t))-y(\beta(t))\right) \\
\leq & a p(t)+b p(\beta(t)) .
\end{aligned}
$$

By (4.3) and Lemma 1, we have for all $t \in[\tau, \infty), p(t) \leq 0$, i.e., $\underline{\alpha}_{n+1} \leq$ $y(t)$. Similarly, we can prove $y(t) \leq \bar{\alpha}_{n+1}, t \in[\tau, \infty)$. Thus $\underline{\alpha}_{n+1} \leq y(t) \leq$ $\bar{\alpha}_{n+1}$, for all $t \in[\tau, \infty)$, which implies $\underline{\alpha}_{*}(t) \leq y(t) \leq \bar{\alpha}_{*}(t)$. We complete the proof.

\section{Conclusions}

In this paper, the monotone iterative technique is used to solve first-order differential equations with piecewise constant generalized delay (2.1) and some new comparison results are obtained. Two converging monotone sequences are obtained with the monotone iterative technique based on upper and lower solutions. Those two converging monotone sequences will converge to the extremal solution of the DEPCAG (2.1).

\section{Acknowledgements}

Author's research was supported in part by PGI 03-2020 DIUMCE.

\section{References}

[1] A. R. Aftabizadeh and J. Wiener, "Differential inequalities for delay differential equations with piecewise constant argument", Applied mathematics computations, vol. 24, no. 3, pp. 183-194, Dec. 1987, doi: 10.1016/0096-3003(87)90083-X

[2] M. U. Akhmet, "Integral manifolds of differential equations with piecewise constant argument of generalized type", Nonlinear analysis: theory, methods \& applications, vol. 66, no. 2, pp. 367-383, Jan. 2007, doi: $10.1016 /$ j.na.2005.11.032 
[3] S. Busenberg and K. Cooke, Vertically transmitted diseases models and dynamics. Berlin: Springer, 1993, doi: 10.1007/978-3-64275301-5

[4] K. L. Cooke and J. Wiener, "Retarded differential equations with piecewise constant delays", Journal mathematics analysis applications, vol. 99, no. 1, pp. 265-297, Mar. 1984, doi: $10.1016 / 0022-247 X(84) 90248-8$

[5] K.-S. Chiu and M. Pinto, "Periodic solutions of differential equations with a general piecewise constant argument and applications", Electronic journal qualitative theory of differential equations, vol. 46, Art ID. 46, 2010, doi: 10.14232/ ejqtde.2010.1.46

[6] K.-S. Chiu and M. Pinto, "Variation of parameters formula and Gronwall inequality for differential equations with a general piecewise constant argument”, Acta Mathematicae Applicatae Sinica, English Series, vol. 27, Art ID. 561, Sep. 2011, doi: 10.1007/s 10255-011-0107-5

[7] K.-S. Chiu and M. Pinto, "Oscillatory and periodic solutions in alternately advanced and delayed differential equations", Carpathian journal mathematics, vol. 29, no. 2, pp. 149-158, 2013. [On line]. Available: https:// bit.ly/3qc9GrG

[8] K.-S. Chiu, "Periodic solutions for nonlinear integro-differential systems with piecewise constant argument", The scientific world journal, vol. 2014, Art ID. 514854, Jan. 2014, doi:10.1155/2014/ 514854

[9] K.-S. Chiu, M. Pinto, and J.-Ch. Jeng, "Existence and global convergence of periodic solutions in recurrent neural network models with a general piecewise alternately advanced and retarded argument", Acta applicandae mathematicae, vol. 133, pp. 133-152, Oct. 2014, doi: $10.1007 / \mathrm{s} 10440-013-9863-\mathrm{y}$

[10] K.-S. Chiu and J.-Ch. Jeng, "Stability of oscillatory solutions of differential equations with general piecewise constant arguments of mixed type", Mathematische nachrichten, vol. 288, no. 10, pp. 1085-1097, Jul. 2015, doi: 10.1002/ mana.201300127

[11] K.-S. Chiu, "On generalized impulsive piecewise constant delay differential equations", Science China Mathematics, vol. 58, pp. 1981-2002, Sep. 2015, doi: 10.1007/s11425-015-5010-8

[12] K.-S. Chiu, "Exponential stability and periodic solutions of impulsive neural network models with piecewise constant argument", Acta applied mathematics, vol. 151, pp. 199-226, Oct. 2017, doi: 10.1007/s 10440-017-0108-3 
[13] K.-S. Chiu, "Asymptotic equivalence of alternately advanced and delayed differential systems with piecewise constant generalized arguments", Acta mathematical sciences, vol. 38, no. 1, pp. 220-236, Jan. 2018, doi: 10.1016/ S0252-9602(17)30128-5

[14] K.-S. Chiu and T. Li, "Oscillatory and periodic solutions of differential equations with piecewise constant generalized mixed arguments", Mathematische nachrichten, vol. 292, no. 10, pp. 2153-2164, Oct. 2019, doi: 10.1002/ mana.201800053

[15] J. Gouzé and T. Sari, "A class of piecewise linear differential equations arising in biological models", Dynamics systems, vol. 17, no. 4, pp. 299-316, 2002, doi: 10.1080/1468936021000041681

[16] T. Küpper and R. Yuang, "On quasi-periodic solutions of differential equations with piecewise constant argument", Journal mathematics analysis applications, vol. 267, no. 1, pp. 173-193, Mar. 2002, doi: $10.1006 /$ jmaa.2001.7761

[17] G. S Ladde, V. Lakshmikantham, and A. S. Vatsala, Monotone iterative techniques for nonlinear differential equations. Boston, MA: Pitman, Boston, 1985.

[18] V. Lakshmikantham and S. Leela, Eds., Differential and integral inequalities: theory and applications, 2 vols. New York, NY: Academic Press, 1969.

[19] M. Pinto, "Asymptotic equivalence of nonlinear and quasilinear differential equations with piecewise constant arguments", Mathematics computation modelating, vol. 49, no. 9-10, pp. 1750-1758, May 2009, doi: $10.1016 /$ j.mcm.2008.10.001

[20] S. M. Shah and J. Wiener, "Advanced differential equations with piecewise constant argument deviations", International journal mathematical of sciences, vol. 6, no. 4, pp. 671-703, 1983, doi: $10.1155 /$ S0161171283000599

[21] W. Walter, Differential and integral inequalities. Berlin: Springer, 1970, doi: 10.1007/978-3-642-86405-6

[22] J. Wiener, Generalized solutions of functional differential equations. Singapore: World Scientific, 1993, doi: 10.1142/ 1860

[23] F. Zhang, A. Zhao, and J. Yan, "Monotone iterative method for differential equations with piecewise constant arguments", Portugaliae mathematica, vol. 57, no. 3, pp. 345-353, 2000. [On line]. Available: https:// bit.ly/2XCSWxs 